\title{
Synthesis, structure and electrochemical properties of some cobalt oxalates
}

Jinghua Cai, ${ }^{1+}$ Yuanqi Lan, ${ }^{1,2}$ Haiyan He, ${ }^{1+}$ Xinyuan Zhang, ${ }^{4 *}$ A. Robert Armstrong, ${ }^{5}$ Wenjiao Yao, ${ }^{*}$ Philip Lightfoot, ${ }^{5 *}$ Yongbing Tang ${ }^{1,2,3 *}$

${ }^{1}$ Functional Thin Films Research Center, Shenzhen Institutes of Advanced Technology, Chinese Academy of Sciences, Shenzhen 518055, China.

${ }^{2}$ Shenzhen College of Advanced Technology, University of Chinese Academy of Sciences, Shenzhen 518055, China.

${ }^{3}$ Key Laboratory of Advanced Materials Processing \& Mold, Ministry of Education, Zhengzhou University, Zhengzhou 450002, China

4 Tianjin Key Laboratory of Functional Crystal Materials, Institute of Functional Crystals, Tianjin University of Technology, Tianjin 300384, China.

${ }^{5}$ School of Chemistry, University of St Andrews, St Andrews, Fife KY16 9ST, UK.

\section{Corresponding Author}

wj.yao@siat.ac.cn (W. Yao)

pl@st-andrews.ac.uk (P. Lightfoot)

tangyb@siat.ac.cn (Y. Tang)

KEYWORDS: Cobalt-based oxalate, synthesis, structure, electrochemical properties. 


\section{Table of Content}

1. Atomic parameters for $\mathrm{Li}_{4} \mathrm{Co}\left(\mathrm{C}_{2} \mathrm{O}_{4}\right)_{3}$

2. Key bond lengths in $\mathrm{Li}_{4} \mathrm{Co}\left(\mathrm{C}_{2} \mathrm{O}_{4}\right)_{3}$

3. Atomic parameters for $\left(\mathrm{NH}_{4}\right)_{2} \mathrm{Co}_{2}\left(\mathrm{C}_{2} \mathrm{O}_{4}\right) \mathrm{F}_{4}$

4. Key bond lengths in $\left(\mathrm{NH}_{4}\right)_{2} \mathrm{Co}_{2}\left(\mathrm{C}_{2} \mathrm{O}_{4}\right) \mathrm{F}_{4}$

5. Schematic structures of $\mathrm{Na}_{2} \mathrm{Co}_{2}\left(\mathrm{C}_{2} \mathrm{O}_{4}\right)_{3} \cdot 2 \mathrm{H}_{2} \mathrm{O}(\boldsymbol{I})$

6. Schematic structures of $\mathrm{Na}_{2} \mathrm{Co}\left(\mathrm{C}_{2} \mathrm{O}_{4}\right)_{2} \cdot 8 \mathrm{H}_{2} \mathrm{O}($ II $)$

7. Schematic structures of $\mathrm{KLi}_{3} \mathrm{Co}\left(\mathrm{C}_{2} \mathrm{O}_{4}\right)_{3}$ (III)

8. Attemptive synthesis conditions of $\mathrm{Li}_{4} \mathrm{Co}\left(\mathrm{C}_{2} \mathrm{O}_{4}\right)_{3}$

9. Characterization of $\left(\mathrm{NH}_{4}\right)_{2} \mathrm{Co}_{2}\left(\mathrm{C}_{2} \mathrm{O}_{4}\right) \mathrm{F}_{4}$

10. A photo image and powder $\mathrm{XRD}$ of the Co- analogue to $\mathrm{KFeC}_{2} \mathrm{O}_{4} \mathrm{~F}$

11. Powder XRD of ball-milled $\boldsymbol{I}$ and the first charge-discharge curve

12. Powder XRD of ball-milled $\boldsymbol{I I I}$ and the first charge-discharge curve 
Table S1 Atomic parameters in $\mathrm{Li}_{4} \mathrm{Co}\left(\mathrm{C}_{2} \mathrm{O}_{4}\right)_{3}$ (space group $P 2_{1} / a, a=9.3619(7) \AA, b$ $\left.=5.0589(3) \AA, c=10.0325(7) \AA, \beta=104.923(7)^{\circ}\right)$

\begin{tabular}{|ccccccc|} 
Atom & Wyck. Site & $\mathbf{x} / \mathbf{a}$ & $\mathbf{y} / \mathbf{b}$ & $\mathbf{z} / \mathbf{c}$ & $\mathbf{U}\left[\mathbf{1 0}^{-2} \AA^{2}\right]$ \\
Co1 & $2 \mathrm{a}$ & -1 & 0 & 0 & 0 & $0.692(14)$ \\
$\mathrm{O} 1$ & $4 \mathrm{e}$ & 1 & $-0.23152(9)$ & $0.20758(16)$ & $0.27707(8)$ & $1.01(2)$ \\
$\mathrm{O} 2$ & $4 \mathrm{e}$ & 1 & $-0.05184(9)$ & $0.20701(15)$ & $0.16597(9)$ & $0.88(2)$ \\
$\mathrm{O} 3$ & $4 \mathrm{e}$ & 1 & $-0.15700(9)$ & $0.25929(16)$ & $-0.14339(8)$ & $1.00(2)$ \\
$\mathrm{O} 4$ & $4 \mathrm{e}$ & 1 & $0.04235(9)$ & $-0.22082(16)$ & $0.38270(9)$ & $1.10(2)$ \\
$\mathrm{O} 5$ & $4 \mathrm{e}$ & 1 & $-0.36473(10)$ & $0.25903(16)$ & $0.50472(9)$ & $1.21(2)$ \\
$\mathrm{O} 6$ & $4 \mathrm{e}$ & 1 & $-0.15681(9)$ & $-0.24217(15)$ & $0.04066(8)$ & $0.89(2)$ \\
$\mathrm{C} 1$ & $4 \mathrm{e}$ & 1 & $-0.22796(12)$ & $-0.1455(2)$ & $0.11890(11)$ & $0.79(2)$ \\
$\mathrm{C} 2$ & $4 \mathrm{e}$ & 1 & $-0.02644(13)$ & $-0.1351(2)$ & $0.46492(13)$ & $0.83(2)$ \\
$\mathrm{C} 3$ & $4 \mathrm{e}$ & 1 & $-0.16604(12)$ & $0.1151(2)$ & $0.19415(12)$ & $0.78(2)$ \\
$\mathrm{Li} 1$ & $4 \mathrm{e}$ & 1 & $-0.2749(4)$ & $0.4994(3)$ & $0.3873(4)$ & $1.69(7)$ \\
$\mathrm{L} 22$ & $4 \mathrm{e}$ & 1 & $0.0748(4)$ & $-0.5073(3)$ & $0.2654(4$ & $1.64(6)$ \\
\hline
\end{tabular}

Table S2 Typical bond lengths in $\mathrm{Li}_{4} \mathrm{Co}\left(\mathrm{C}_{2} \mathrm{O}_{4}\right)_{3}$ (space group $P 2_{1} / a, a=9.3619(7) \AA$,

$$
\left.b=5.0589(3) \AA, c=10.0325(7) \AA, \beta=104.923(7)^{\circ}\right)
$$

\begin{tabular}{|ccc|ccc|}
\hline Bond & Length $(\AA)$ & Multiplicity & Bond & Length $(\AA)$ & Multiplicity \\
\hline Co-O2 & $2.1263(8)$ & 2 & C2-O4 & $1.2477(14)$ & 1 \\
Co-O3 & $2.2048(8)$ & 2 & C2-O5 & $1.256(2)$ & 1 \\
Co-O6 & $2.0325(8)$ & 2 & C3-O1 & $1.2448(14)$ & 1 \\
C1-C3 & $1.555(2)$ & 1 & C3-O2 & $1.2629(14)$ & 1 \\
C2-C2 & $1.559(2)$ & 1 & & & \\
C1-O3 & $1.2605(14)$ & 1 & & & \\
C1-O6 & $1.2523(14)$ & 1 & & & \\
\hline
\end{tabular}


Table S3 Atomic parameters in $\left(\mathrm{NH}_{4}\right)_{2} \mathrm{Co}_{2}\left(\mathrm{C}_{2} \mathrm{O}_{4}\right) \mathrm{F}_{4}$ (space group Cmmm, $a=$

$$
\text { 6.1074(5) } \AA, b=16.3644(14) \AA, c=4.0298(3) \AA)
$$

\begin{tabular}{|ccccccc|}
\multicolumn{2}{|c}{ Atom Wyck. } & Site & $\mathbf{x} / \mathbf{a}$ & $\mathbf{y} / \mathbf{b}$ & $\mathbf{z} / \mathbf{c}$ & $\mathbf{U}\left[\mathbf{1 0}^{-2} \AA^{2}\right]$ \\
\hline $\mathrm{Co} 1$ & $4 \mathrm{j}$ & $\mathrm{m} 2 \mathrm{~m}$ & $1 / 2$ & $0.33243(2)$ & $1 / 2$ & $0.81(2)$ \\
$\mathrm{F} 1$ & $4 \mathrm{f}$ & $. .2 / \mathrm{m}$ & $1 / 4$ & $1 / 4$ & $1 / 2$ & $1.85(4)$ \\
$\mathrm{F} 2$ & $4 \mathrm{i}$ & $\mathrm{m} 2 \mathrm{~m}$ & $1 / 2$ & $0.32950(13)$ & 0 & $2.25(5)$ \\
$\mathrm{O} 1$ & $8 \mathrm{q}$ &.. $\mathrm{m}$ & $0.7202(3)$ & $0.43173(9)$ & $1 / 2$ & $1.33(3)$ \\
$\mathrm{N} 1$ & $4 \mathrm{i}$ & $\mathrm{m} 2 \mathrm{~m}$ & $1 / 2$ & $0.1492(2)$ & 0 & $1.35(6)$ \\
$\mathrm{C} 1$ & $4 \mathrm{~h}$ & $2 \mathrm{~mm}$ & $0.6286(5)$ & $1 / 2$ & $1 / 2$ & $0.87(6)$ \\
$\mathrm{H} 1$ & $8 \mathrm{n}$ & $\mathrm{m} .$. & $1 / 2$ & $0.117(4)$ & $-0.152(16)$ & $0.09(2)$ \\
$\mathrm{H} 2$ & $8 \mathrm{p}$ &.. $\mathrm{m}$ & $0.405(9)$ & $0.173(3)$ & 0 & $0.10(2)$ \\
\hline
\end{tabular}

Table S4 Typical bond lengths in $\left(\mathrm{NH}_{4}\right)_{2} \mathrm{Co}_{2}\left(\mathrm{C}_{2} \mathrm{O}_{4}\right) \mathrm{F}_{4}$ (space group Cmmm, $a=$

$$
\text { 6.1074(5) } \AA, b=16.3644(14) \AA, c=4.0298(3) \AA)
$$

\begin{tabular}{|ccc|ccc|}
\hline Bond & Length $(\AA)$ & Multiplicity & Bond & Length $(\AA)$ & Multiplicity \\
\hline Co-F1 & $2.0374(2)$ & 2 & N1-H1 & $0.70(6)$ & 2 \\
Co-F2 & $2.0155(2)$ & 2 & N1-H2 & $0.81(7)$ & 2 \\
Co-O1 & $2.1095(2)$ & 2 & & & \\
C1-C1 & $1.571(6)$ & 1 & & & \\
C1-O1 & $1.249(2)$ & 2 & & & \\
\hline
\end{tabular}


(a)

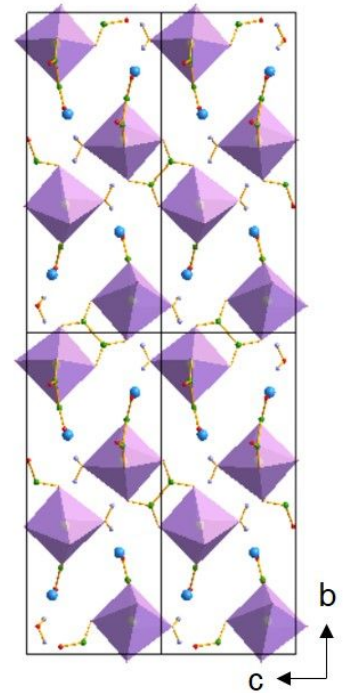

(b)

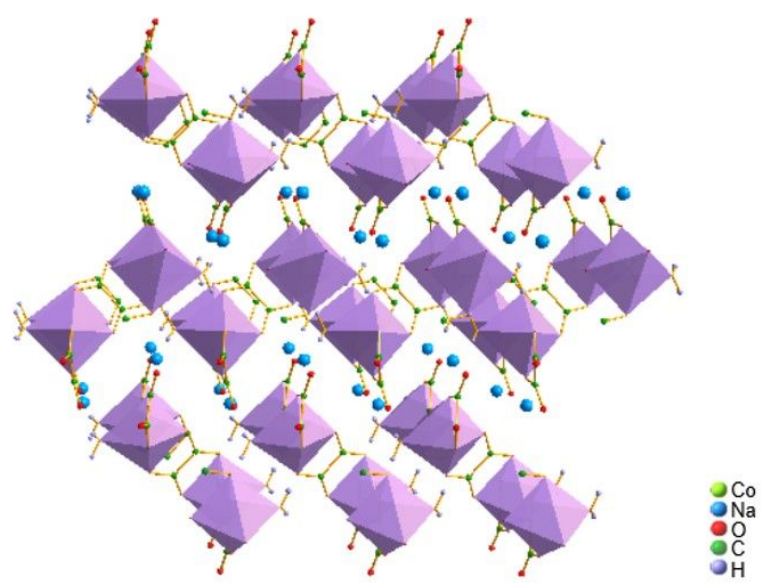

Figure S1 Schematic structure of $\mathrm{Na}_{2} \mathrm{Co}_{2}\left(\mathrm{C}_{2} \mathrm{O}_{4}\right)_{3} \cdot 2 \mathrm{H}_{2} \mathrm{O}$ (I). ICSD 414681, Ref: $J$. Chem. Soc., Dalton Trans. 2000, (20), 3566-3569.

(a)

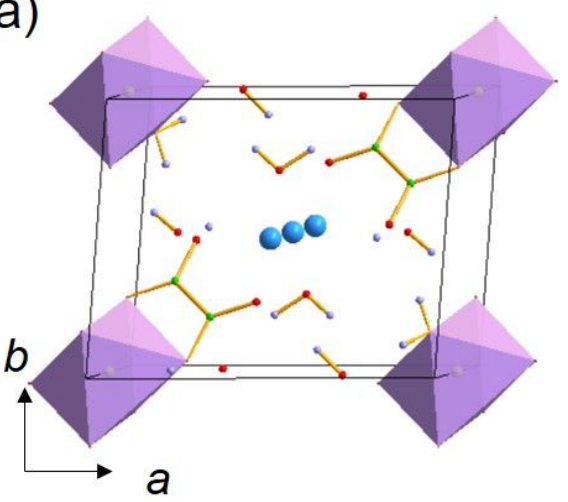

(b)

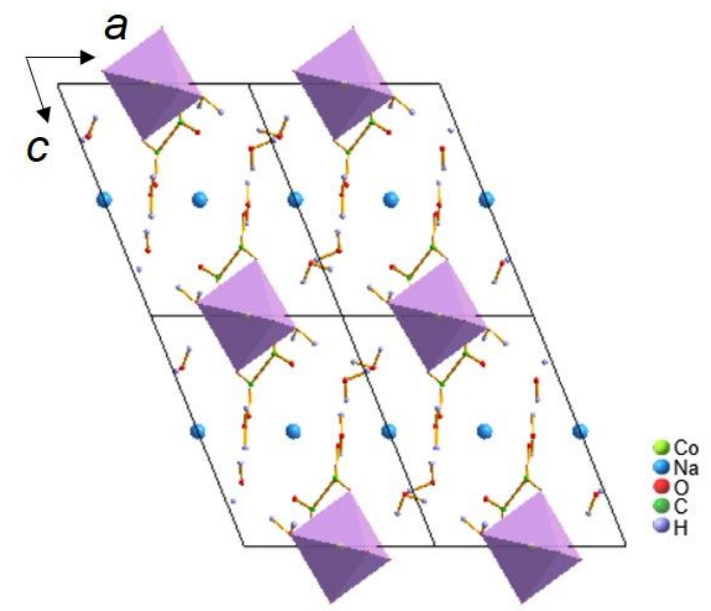

Figure S2 Schematic structure of $\mathrm{Na}_{2} \mathrm{Co}\left(\mathrm{C}_{2} \mathrm{O}_{4}\right)_{2} \cdot 8 \mathrm{H}_{2} \mathrm{O}$ (II). CCDC 1401089, Ref:

Dalton Trans. 2016, 45 (20), 8500-8505. 
(a)

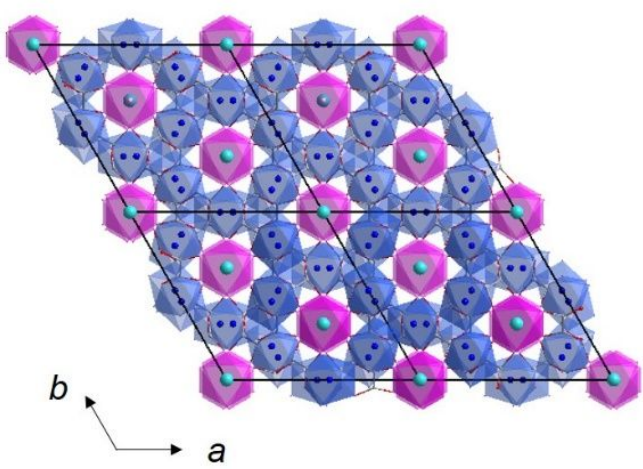

(b)

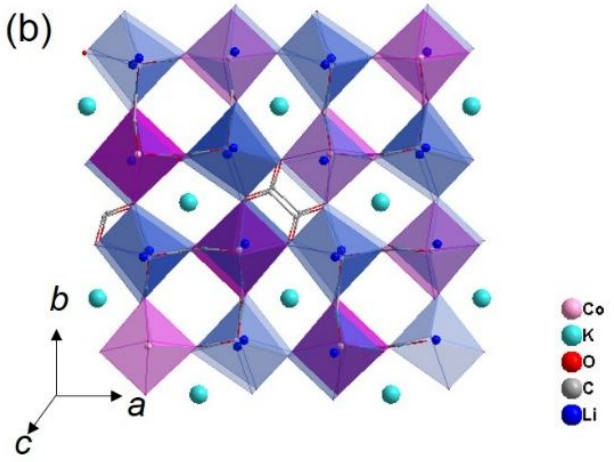

Figure S3 Schematic structure of $\mathrm{KLi}_{3} \mathrm{Co}\left(\mathrm{C}_{2} \mathrm{O}_{4}\right)_{3}$ (III). ICSD 244141, Ref:

Dalton Trans. 2019, 48 (38), 14461-14466. 
Table S5 Attemptive synthesis conditions for $\mathrm{Li}_{4} \mathrm{Co}\left(\mathrm{C}_{2} \mathrm{O}_{4}\right)_{3}$

\begin{tabular}{|c|c|c|c|c|c|c|}
\hline No. & $\begin{array}{c}\mathrm{CoCl}_{2} \cdot 6 \mathrm{H}_{2} \mathrm{O} \\
(\mathrm{mmol})\end{array}$ & $\begin{array}{l}\mathrm{Li}_{2} \mathrm{CO}_{3} \\
(\mathrm{mmol})\end{array}$ & $\begin{array}{c}\mathrm{H}_{2} \mathrm{C}_{2} \mathrm{O}_{4} \cdot 2 \mathrm{H}_{2} \mathrm{O} \\
(\mathrm{mmol})\end{array}$ & $\begin{array}{l}\mathrm{H}_{2} \mathrm{O} \\
(\mathrm{mL})\end{array}$ & $\begin{array}{c}\text { Temperature } \\
\left({ }^{\circ} \mathrm{C}\right)\end{array}$ & $\begin{array}{l}\text { Time } \\
\text { (days) }\end{array}$ \\
\hline 1 & 0.7 & 1.8 & 2.4 & 3 & 160 & 3 \\
\hline 2 & 0.8 & 1.8 & 2.4 & 3 & 160 & 3 \\
\hline 3 & 0.9 & 1.8 & 2.4 & 3 & 160 & 3 \\
\hline 4 & 1.0 & 1.8 & 2.4 & 3 & 160 & 3 \\
\hline 5 & 1.1 & 1.8 & 2.4 & 3 & 160 & 3 \\
\hline 6 & 1.2 & 1.8 & 2.4 & 3 & 160 & 3 \\
\hline 7 & 0.5 & 1.0 & 1.2 & 3 & 190 & 2 \\
\hline 8 & 0.6 & 1.0 & 1.2 & 3 & 190 & 2 \\
\hline 9 & 0.7 & 1.0 & 1.2 & 3 & 190 & 2 \\
\hline 10 & 0.8 & 1.0 & 1.2 & 3 & 190 & 2 \\
\hline 11 & 0.9 & 1.0 & 1.2 & 3 & 190 & 2 \\
\hline 12 & 1.0 & 1.0 & 1.2 & 3 & 190 & 2 \\
\hline 13 & 0.5 & 1.8 & 2.4 & 3 & 160 & 3 \\
\hline 14 & 0.6 & 1.8 & 2.4 & 3 & 160 & 3 \\
\hline 15 & 0.7 & 1.8 & 2.4 & 3 & 160 & 3 \\
\hline 16 & 0.3 & 1.0 & 1.2 & 2 & 160 & 3 \\
\hline 17 & 0.4 & 1.0 & 1.2 & 2 & 160 & 3 \\
\hline 18 & 0.5 & 1.0 & 1.2 & 2 & 160 & 3 \\
\hline 19 & 0.6 & 1.0 & 1.2 & 2 & 160 & 3 \\
\hline 20 & 0.10 & 1.0 & 1.2 & 2 & 160 & 3 \\
\hline 21 & 0.15 & 1.0 & 1.2 & 2 & 160 & 3 \\
\hline 22 & 0.20 & 1.0 & 1.2 & 2 & 160 & 3 \\
\hline 23 & 0.25 & 1.0 & 1.2 & 2 & 160 & 3 \\
\hline 24 & 0.30 & 1.0 & 1.2 & 2 & 160 & 3 \\
\hline 25 & 0.30 & 1.0 & 1.2 & 1 & 160 & 3 \\
\hline 26 & 0.20 & 1.0 & 1.2 & 1 & 160 & 3 \\
\hline 27 & 0.10 & 1.0 & 1.2 & 2 & 180 & 2 \\
\hline 28 & 0.15 & 1.0 & 1.2 & 2 & 180 & 2 \\
\hline
\end{tabular}

None of the above conditions could produce $\mathrm{Li}_{4} \mathrm{Co}\left(\mathrm{C}_{2} \mathrm{O}_{4}\right)_{3}$. 

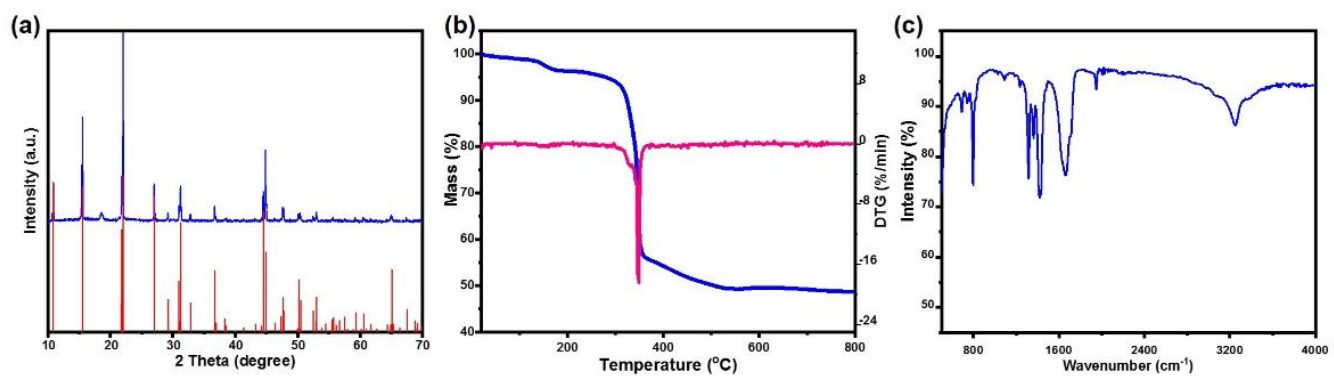

Figure S4 Characterization of $\left(\mathrm{NH}_{4}\right)_{2} \mathrm{Co}_{2}\left(\mathrm{C}_{2} \mathrm{O}_{4}\right) \mathrm{F}_{4}$. (a) Powder XRD using a Rigaku diffractometer (MiniFlex600). The peak at $\sim 18.7^{\circ}$ in XRD comes from trace amount of cobalt oxalate dihydrate. (b) Thermogravimetric and differential thermogravimetric curves in $\mathrm{N}_{2}$ flow using a STA449F3 thermal analyzer. (c) Infrared vibration pattern using a Perkin Elmer Frontier.

(a)

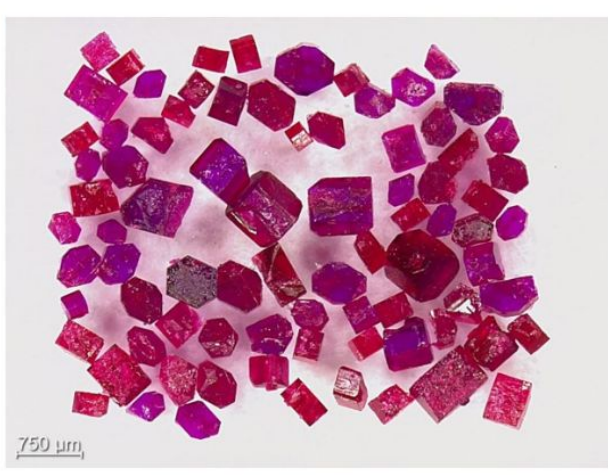

(b)

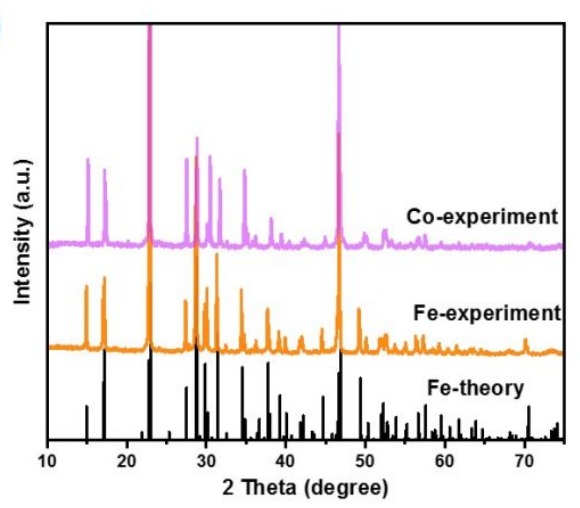

Figure S5 A photo image and powder XRD of Co- analogue to $\mathrm{KFeC}_{2} \mathrm{O}_{4} \mathrm{~F}$. 
(a)

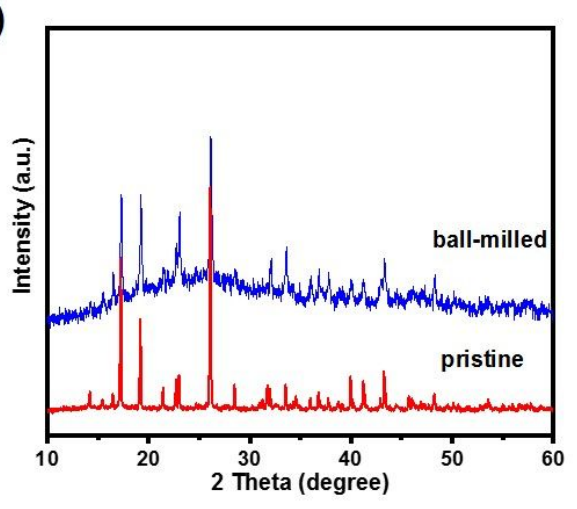

(b)

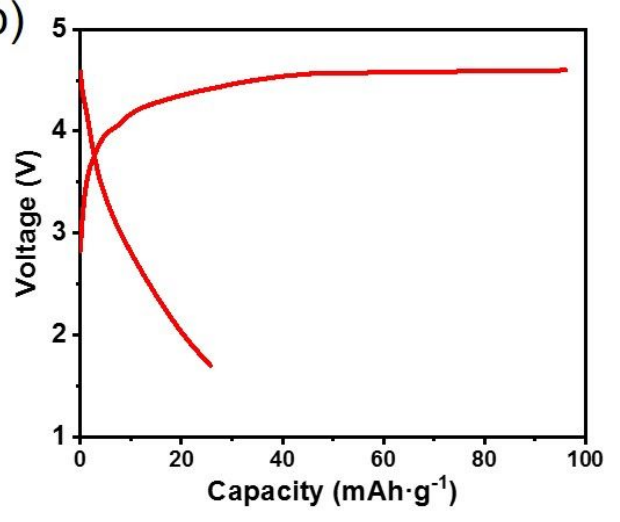

Figure S6 Powder XRD of ball-milled $\boldsymbol{I}$ and the first charging-discharging curve.

(a)

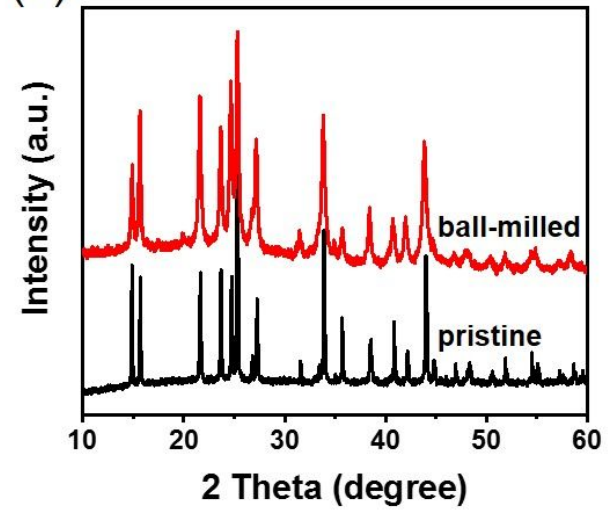

(b)

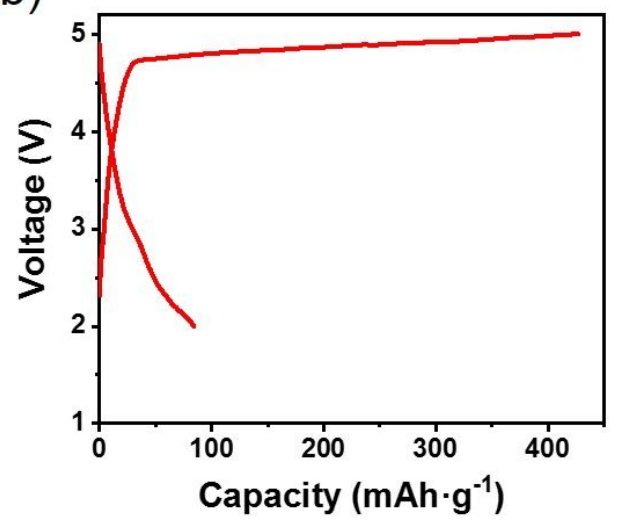

Figure S7 Powder XRD of ball-milled $\boldsymbol{I I I}$ and the first charging-discharging curve. 